\title{
Energy-Efficient Wireless Network Design ${ }^{\star}$
}

\author{
Ioannis Caragiannis, Christos Kaklamanis, and Panagiotis Kanellopoulos \\ Computer Technology Institute and \\ Dept. of Computer Engineering and Informatics \\ University of Patras, 26500 Rio, Greece
}

\begin{abstract}
A crucial issue in ad hoc wireless networks is to efficiently support communication patterns that are typical in traditional (wired) networks. These include broadcasting, multicasting, and gossiping (allto-all communication). Since, in ad hoc networks energy is a scarce resource, the important engineering question to be solved is to guarantee a desired communication pattern minimizing the total energy consumption. Motivated by this question, we study a series of wireless network design problems and present new approximation algorithms and inapproximability results.
\end{abstract}

\section{Introduction}

Wireless networks have received significant attention during the recent years. Especially, ad hoc wireless networks emerged due to their potential applications in battlefield, emergency disaster relief, etc. [16]. Unlike traditional wired networks or cellular wireless networks, no wired backbone infrastructure is installed for ad hoc wireless networks.

A node in these networks is equipped with an omnidirectional antenna which is responsible for sending and receiving signals. Communication is established by assigning to each station a transmitting power. In the most common power attenuation model [16], the signal power falls as $1 / r^{\alpha}$, where $r$ is the distance from the transmitter and $\alpha$ is a constant which depends on the wireless environment (typical values of $\alpha$ are between 1 and 6 ). So, a transmitter can send a signal to a receiver if $\frac{P_{s}}{d(s, t)^{\alpha}} \geq \gamma$ where $P_{s}$ is the power of the transmitting signal, $d(s, t)$ is the Euclidean distance between the transmitter and the receiver, and $\gamma$ is the receiver's power threshold for signal detection.

So, communication from a node $s$ to another node $t$ may be established either directly if the two nodes are close enough and $s$ uses adequate transmitting power, or by using intermediate nodes. Observe that due to the nonlinear power attenuation, relaying the signal between intermediate nodes may result in energy conservation.

A crucial issue in ad hoc wireless networks is to support communication patterns that are typical in traditional networks. These include broadcasting, multicasting, and gossiping (all-to-all communication). Since establishing a communication pattern strongly depends on the use of energy, the important engineering

\footnotetext{
* This work was partially supported by the European Union under IST FET Project ALCOM-FT, IST FET Project CRESCCO, and RTN Project ARACNE.
} 
question to be solved is to guarantee a desired communication pattern minimizing the total energy consumption. In this work, we consider a series of wireless network design problems which we formulate below.

Consider a complete directed graph $G=(V, E)$, where $|V|=n$, with a nonnegative edge cost function $c: E \rightarrow R^{+}$. Given a non-negative node weight assignment $w: V \rightarrow R^{+}$, the transmission graph $G_{w}$ is the directed graph defined as follows. It has the same set of nodes as $G$ and a directed edge $(u, v)$ belongs to $G_{w}$ if the weight assigned to node $u$ is at least the cost of the edge $(u, v)$, i.e., $w(u) \geq c(u, v)$. Intuitively, the weight assignment corresponds to the energy levels at which each node operates (i.e., transmits messages) while the cost between two nodes indicates the minimum energy level necessary to send messages from one node to the other. Usually, the edge cost function is symmetric (i.e., $c(u, v)=c(v, u))$. Asymmetric edge cost functions can be used to model medium abnormalities or batteries with different energy levels [14].

The problems we study can be stated as follows. Given a complete directed graph $G=(V, E)$ with $|V|=n$, with non-negative edge $\operatorname{costs} c: E \rightarrow R^{+}$, find a non-negative node weight assignment $w: V \rightarrow R^{+}$such that the transmission graph $G_{w}$ maintains a connectivity property and the sum of weights is minimized. Such a property is defined by a requirement matrix $R=\left(r_{i j}\right) \in\{0,1\}$ where $r_{i j}$ is the number of directed paths required in the transmission graph from node $v_{i}$ to node $v_{j}$. Depending on the connectivity property for the transmission graph, we may define the following problems.

In Minimum Energy Steiner Subgraph (MESS), the requirement matrix is symmetric. Alternatively, we may define the problem by a set of nodes $D \subseteq V$ partitioned into $p$ disjoint subsets $D_{1}, D_{2}, \ldots, D_{p}$. The entries of the requirement matrix are now defined as $r_{i j}=1$ if $v_{i}, v_{j} \in D_{k}$ for some $k$ and $r_{i j}=0$, otherwise. The Minimum Energy Subset Strongly Connected SUbGRAPH (MESSCS) is the special case of MESS with $p=1$ while the MinImum Energy Strongly Connected Subgraph (MESCS) is the special case of MESSCS with $D=V$ (i.e., the transmission graph is required to span all nodes of $V$ and to be strongly connected). The authors of [1] study MESCS under the extra requirement that the transmission graph contains a bidirected subgraph which maintains the connectivity requirements of MESCS. By adding this extra requirement to MESS and MESSCS, we obtain the bidirected MESS and bidirected MESSCS, respectively. For bidirected MESCS, [1] shows a constant approximation algorithm in symmetric graphs and a logarithmic inapproximability result in asymmetric graphs. The same reduction used to prove this inapproximability result can be used for proving a logarithmic inapproximability result for MESCS in asymmetric graphs as well. MESCS in symmetric graphs is studied by Kirousis et al. [13] who present (among other results) a 2-approximation algorithm. Clementi et al. [8] study geometric versions of the problem and show approximation-preserving reductions from VERTEX COVER on bounded-degree graphs to geometric instances of MESCS. By adapting the reduction of [8] and using the hardness results of [2], we can obtain an inapproximability factor of 313/312 for MESCS in symmetric graphs. As observed in [1], this result holds for bidirected MESCS as well. 
In Minimum Energy Multicast Tree (MEMT), the connectivity property is defined by a root node $v_{0}$ and a set of nodes $D \subseteq V-\left\{v_{0}\right\}$ such that $r_{i j}=1$ if $i=0$ and $v_{j} \in D$ and $r_{i j}=0$, otherwise. The Minimum Energy Broadcast Tree (MEBT) is the special case of MEMT with $D=V-\left\{v_{0}\right\}$. Liang [14] shows an $O\left(|D|^{\epsilon}\right)$ - and an $O\left(n^{\epsilon}\right)$-approximation algorithm for MEMT and MEBT, respectively, for any constant $\epsilon>0$. These results follow by using an intuitive reduction of any instance of MEMT to an instance of DIRECTED STEINER TREE and then applying the algorithm of Charikar et al. [5] for computing an approximate directed Steiner tree which gives an approximate solution to MEMT. Note that the work of Liang does not answer the question whether MEMT and MEBT are strictly easier to approximate than DiRECTED STEINER TREE or not. For MEMT in symmetric graphs, Liang 14 shows an $O\left(\ln ^{3}|D|\right)$ approximation algorithm while, for MEBT in symmetric graphs, we show in [4] a $10.8 \ln n$-approximation algorithm by reducing instances of the problem to instances of Node-Weighted Connected Dominating Set and using the algorithm of 11] for computing an approximate connected dominating set which gives an approximate solution to MEBT. Constant approximation algorithms for geometric versions of MEBT are presented in [7]16].

By inverting the connectivity requirements, we obtain the MINIMUM ENERGy Inverse Multicast Tree (MEIMT) where the connectivity property is defined by a root node $v_{0}$ and a set of nodes $D \subseteq V-\left\{v_{0}\right\}$ such that $r_{i j}=1$ if $v_{i} \in D$ and $j=0$ and $r_{i j}=0$, otherwise, and the Minimum ENERGy InVERSE BroAdCAst TREE (MEIBT) which is the special case of MEIMT with $D=V-\left\{v_{0}\right\}$.

In the paper, we usually refer to classical combinatorial optimization problems. For completeness, we present their definitions here. The STEINER ForEst (SF) problem is defined as follows. Given an undirected graph $G=(V, E)$ with an edge cost function $c: E \rightarrow R^{+}$and a set of nodes $D \subseteq V$ partitioned into $p$ disjoint sets $D_{1}, \ldots, D_{p}$, compute a subgraph $H$ of $G$ of minimum total cost such that any two nodes $v_{i}, v_{j}$ belonging to the same set $D_{k}$ for some $k$ are connected through a path in $H$. STEINER TREE (ST) is the special case of SF with $p=1$. An instance of the Directed Steiner Tree (DST) is defined by a directed graph $G=(V, E)$ with an edge cost function $c: E \rightarrow R^{+}$, a root node $v_{0} \in V$, and a set of terminals $D \subseteq V-\left\{v_{0}\right\}$. Its objective is to compute a tree of minimum cost which is directed out of $v_{0}$ and spans all nodes of $D$. The NodEWeighted Steiner Forest (NWSF) problem is defined as follows. Given an undirected graph $G=(V, E)$ with a node cost function $c: V \rightarrow R^{+}$and a set of nodes $D \subseteq V$ partitioned into $p$ disjoint sets $D_{1}, \ldots, D_{p}$, compute a subgraph $H$ of $G$ of minimum total cost such that any two nodes $v_{i}, v_{j}$ belonging to the same set $D_{k}$ for some $k$ are connected through a path in $H$. NoDE-WEIGHTED Steiner TREe (NWST) is the special case of NWSF with $p=1$.

In the rest of this section we summarize our results. In Section 2 we present constant approximation algorithms for MESS, MESSCS, bidirected MESS and bidirected MESSCS in symmetric graphs (i.e., input instances with symmetric edge cost functions) exploiting known efficient approximation algorithms for SF and ST. For bidirected MESSCS, we also give a simple inapproximability result. 
We also present approximation algorithms for MEMT and MEBT in symmetric graphs (Section 3) with logarithmic approximation ratios by using a new reduction of instances of MEMT and MEBT to instances of NWST. These results are asymptotically optimal since MEBT in symmetric graphs has been proved to be inapproximable within a sublogarithmic factor 7]. Our result for MEMT improves the polylogarithmic approximation algorithm of Liang 14] while the result for MEBT improves the result of [4] by a multiplicative factor of 4 .

In Section 4, we observe that MEIMT is equivalent to DST, while in Section 5 we show that, in asymmetric graphs, MEMT and MESSCS are at least as hard to approximate as DST. Using a recent inapproximability result for DST due to Halperin and Krauthgamer [12] we obtain polylogarithmic inapproximability results for these problems. On the positive side, we show that MESSCS in asymmetric graphs can be solved by solving an instance of MEMT and an instance of MEIMT.

For MEBT and MESCS in asymmetric graphs, we present logarithmic approximation algorithms exploiting a recent result of Zosin and Khuller [17]. These results are asymptotically optimal. In particular, the result for MEBT significantly improves the $O\left(n^{\epsilon}\right)$-approximation algorithm due to Liang [14. These results are presented in Section 6. In Section 7 we present logarithmic approximation algorithm for bidirected MESS, bidirected MESSCS and bidirected MESCS in asymmetric graphs by using a new reduction of instances of these problems to instances of NWSF. These results asymptotically match the inapproximability result for bidirected MESCS of Althaus et al. 1. Very recently, Gruia Calinescu informed us that logarithmic approximation algorithms for MEBT, MESCS and bidirected MESCS in asymmetric graphs were independently obtained in [3] using different techniques.

Due to lack of space, formal proofs have been omitted from this extended abstract.

\section{Symmetric Graphs and Connectivity Requirements}

In this section, we first show constant approximation algorithms for MESS, MESSCS, bidirected MESS, and bidirected MESSCS, extending the algorithm of Kirousis et al. 13. for MESCS in symmetric graphs. Then, we show an inapproximability result for bidirected MESSCS.

Consider an instance $I_{M E S S}$ of MESS which consists of a complete directed graph $G=(V, E)$, a symmetric edge cost function $c: E \rightarrow R^{+}$and a set of terminals $D \subseteq V$ partitioned into $p$ disjoint subsets $D_{1}, \ldots, D_{p}$. We construct the instance $I_{S F}$ of SF which consists of the complete undirected graph $H=\left(V, E^{\prime}\right)$, the edge cost function $c^{\prime}: E^{\prime} \rightarrow R^{+}$defined as $c^{\prime}(u, v)=c(u, v)=c(v, u)$ on the undirected edges of $E^{\prime}$, and the set of terminals $D$ together with its partition into the sets $D_{1}, \ldots, D_{p}$. Consider a solution for $I_{S F}$ that consists of a subgraph $F=$ $(V, A)$ of $H$. We construct the weight assignment $w$ to the nodes of $V$ by setting $w(u)=0$ if there is no edge touching $u$ in $A$ and $w(u)=\max _{v:(u, v) \in A}\left\{c^{\prime}(u, v)\right\}$, otherwise. We can prove that if $F$ is a $\rho$-approximate solution for $I_{S F}$ then $w$ is a $2 \rho$-approximate solution for $I_{M E S S}$. 
We can solve $I_{S F}$ using the 2-approximation algorithm of Goemans and Williamson 10] for SF. When $p=1$ (i.e., when $I_{M E S S}$ is actually an instance of MESSCS), the instance $I_{S F}$ is actually an instance of ST which can be approximated within 1.55 using an algorithm from [15. We obtain the following.

Theorem 1. There exist a 4- and a 3.1-approximation algorithm for MESS and MESSCS in symmetric graphs, respectively.

Note that the transmission graph constructed by the technique in this section contains a bidirected subgraph that maintains the connectivity requirements of MESS and, thus, our algorithms for MESS and MESSCS provide solutions to bidirected MESS and bidirected MESSCS, respectively. It can be easily seen that the analysis still holds if we consider instances of bidirected MESS and bidirected MESSCS instead of instances of MESS and MESSCS, respectively. Thus, the approximation guarantees of Theorem 1 hold for bidirected MESS and bidirected MESSCS in symmetric graphs as well.

The inapproximability result of 313/312 for MESCS mentioned in the introduction holds for bidirected MESCS in symmetric graphs as well, and, hence, it holds for bidirected MESSCS. We can show a simple approximation-preserving reduction from ST to bidirected MESSCS in symmetric graphs and, using the inapproximability result of [6], we obtain the following.

Theorem 2. For any $\epsilon>0$, bidirected MESSCS is not approximable within $96 / 95-\epsilon$, unless $P=N P$.

\section{Multicasting and Broadcasting in Symmetric Graphs}

In this section we present logarithmic approximation algorithms for MEMT and MEBT in symmetric graphs.

Consider an instance $I_{M E M T}$ of MEMT which consists of a complete directed graph $G=(V, E)$, a symmetric edge cost function $c: E \rightarrow R^{+}$, a root node $v_{0} \in V$ and a set of terminals $D \subseteq V-\left\{v_{0}\right\}$.

We construct an instance $I_{N W S T}$ of NWST, which consists of an undirected graph $H=(U, A)$, a node weight function $c^{\prime}: U \rightarrow R^{+}$and a set of terminals $D^{\prime} \subseteq U$. For a node $v \in V$, we denote by $n_{v}$ the number of different edge costs in the edges directed out of $v$, and, for $i=1, \ldots, n_{v}$, we denote by $X_{i}(v)$ the $i$-th smallest edge cost among the edges directed out of $v$. The set of nodes $U$ consists of $n$ disjoint sets of nodes called supernodes. Each supernode corresponds to a node of $V$. The supernode $Z_{v}$ corresponding to node $v \in V$ has the following $n_{v}+1$ nodes: an input node $Z_{v, 0}$ and $n_{v}$ output nodes $Z_{v, 1}, \ldots, Z_{v, n_{v}}$. For each pair of nodes $u, v \in V$, the set of edges $A$ contains an edge between the output node $Z_{u, i}$ and the input node $Z_{v, 0}$ such that $X_{i}(u) \geq c(u, v)$. Also, for each node $v \in V, A$ contains an edge between the input node $Z_{v, 0}$ and each output node $Z_{v, i}$, for $i=1, \ldots, n_{v}$. The cost function $c^{\prime}$ is defined as $c^{\prime}\left(Z_{v, 0}\right)=0$ for the input nodes and as $c^{\prime}\left(Z_{v, i}\right)=X_{i}(v)$ for $i=1, \ldots, n_{v}$, for the output nodes. The set of terminals $D^{\prime}$ is defined as $D^{\prime}=\left\{Z_{v, 0} \in U \mid v \in D \cup\left\{v_{0}\right\}\right\}$. 
Consider a subgraph $F=\left(S, A^{\prime}\right)$ of $H$ which is a solution for $I_{N W S T}$. We compute a spanning tree $T^{\prime}=\left(S, A^{\prime \prime}\right)$ of $F$ and, starting from $Z_{v_{0}, 0}$, we compute a Breadth First Search (BFS) numbering of the nodes of $T^{\prime}$. For each $v \in S$, we denote by $m(v)$ the BFS number of $v$. We construct a tree $T=\left(V, E^{\prime}\right)$ which, for each edge of $F$ between a node $Z_{u, i}$ of supernode $Z_{u}$ and a node $Z_{v, j}$ of another supernode $Z_{v}$ such that $m\left(Z_{u, i}\right)<m\left(Z_{v, j}\right)$, contains a directed edge from $u$ to $v$. The output of our algorithm is the weight assignment $w$ defined as $w(u)=\max _{(u, v) \in T} c(u, v)$ if $u$ has at least one outgoing edge in $T$, and $w(u)=0$, otherwise. We can prove that if $F$ is a $\rho$-approximate solution to $I_{N W S T}$, then $w$ is a $2 \rho$-approximate solution to $I_{M E M T}$.

In [11, Guha and Khuller present a $1.35 \ln k$-approximation algorithm for NWST, where $k$ is the number of terminals in the instance of NWST. Given an instance $I_{M E M T}$ of MEMT with a set of terminals $D$, the corresponding instance $I_{N W S T}$ has $|D|+1$ terminals. Thus, the cost of the solution of $I_{M E M T}$ is within $2.7 \ln (|D|+1)$ of the optimal solution. The next theorem summarizes the discussion of this section. Note that MEBT is the special case of MEMT with $D=V-\left\{v_{0}\right\}$.

Theorem 3. There exists a $2.7 \ln (|D|+1)$ - and a $2.7 \ln n$-approximation algorithm for MEMT and MEBT in symmetric graphs, respectively.

\section{Approximating MEIMT and MEIBT}

In this section, we first show that MEIMT is equivalent to DST. Assume we have an instance $I_{M E I M T}$ of MEIMT defined by a complete directed graph $G=(V, E)$, an edge cost function $c: E \rightarrow R^{+}$, a root node $v_{0} \in V$ and a set of terminals $D \subseteq V-\left\{v_{0}\right\}$. Consider the instance $I_{D S T}$ of DST that consists of $G$, the edge cost function $c^{\prime}: E \rightarrow R^{+}$defined as $c^{\prime}(u, v)=c(v, u)$ for any edge $(u, v) \in E$, the set of terminals $D$ and the root node $v_{0}$. Also, we may start by an instance $I_{D S T}$ of DST and construct $I_{M E I M T}$ in the same way. We can prove that a $\rho$-approximate solution for $I_{D S T}$ reduces in polynomial time to a $\rho$-approximate solution for $I_{M E I M T}$ and a $\rho$-approximate solution for $I_{M E I M T}$ reduces in polynomial time to a $\rho$-approximate solution for $I_{D S T}$.

Thus, using the results of [5] and [12, we obtain that MEIMT is approximable within $O\left(|D|^{\epsilon}\right)$ and inapproximable within $O\left(\ln ^{2-\epsilon} n\right)$, for any constant $\epsilon>$ 0. Notice that DST in symmetric graphs is equivalent to ST. Thus, using the approximability and inapproximability results of [15] and [6], we obtain that MEIMT in symmetric graphs is approximable within 1.55 and inapproximable within 96/95- $\epsilon$ for any $\epsilon>0$. Also, instances of DST having all non-root nodes as terminals are actually instances of Minimum SPANning ARBorescence which is known to be computable in polynomial time [9]. Thus, MEIBT can be solved in polynomial time (even in asymmetric graphs).

\section{Approximating MEMT and MESSCS}

In this section, we first show that MEMT and MESSCS are as hard to approximate as DST. Then, we present a method for approximating MESSCS. 
Consider an instance $I_{D S T}$ of DST that consists of a directed graph $G=$ $(V, E)$ with an edge cost function $c: E \rightarrow R^{+}$, a root node $v_{0}$ and a set of terminals $D \subseteq V-\left\{v_{0}\right\}$. Without loss of generality, we may assume that $G$ is a complete directed graph with some of its edges having infinite cost.

We construct the instance $I_{M E M T}$ of MEMT which consists of a complete directed graph $H=(U, A)$ with edge cost function $c^{\prime}: A \rightarrow R^{+}$, a root node $v_{0}^{\prime} \in U$ and a set of terminals $D^{\prime} \subseteq U-\left\{v_{0}^{\prime}\right\}$. The set of nodes $U$ has a node $h_{v}$ for each node $v \in V$ and a node $h_{(u, v)}$ for each directed edge $(u, v)$ of $E$. For each directed edge $(u, v)$ of $E$, the directed edge $\left(h_{u}, h_{(u, v)}\right)$ of $A$ has zero cost and the directed edge $\left(h_{(u, v)}, h_{v}\right)$ of $A$ has cost $c^{\prime}\left(h_{(u, v)}, h_{v}\right)=c(u, v)$, while all other edges of $A$ have infinite cost. The set of terminals is defined as $D^{\prime}=\left\{h_{u} \in U \mid u \in D\right\}$, while $v_{0}^{\prime}=h_{v_{0}}$. We can show that a $\rho$-approximate solution to $I_{M E M T}$ reduces in polynomial time to a $\rho$-approximate solution to $I_{D S T}$.

We construct the instance $I_{M E S S C S}$ of MESSCS which consists of the graph $G$, the set of terminals $D$, the root node $v_{0}$, and an edge cost function $c^{\prime \prime}: E \rightarrow$ $R^{+}$defined as follows. For each directed edge $(u, v)$ of $E$ such that $u \neq v_{0}$, it is $c^{\prime \prime}(u, v)=c(v, u)$, while all edges of $E$ directed out of $v_{0}$ have zero cost. We can show that a $\rho$-approximate solution to $I_{M E S S C S}$ reduces in polynomial time to a $\rho$-approximate solution to $I_{D S T}$.

Using the inapproximability result for DST [12, we obtain the following.

Theorem 4. For any $\epsilon>0, M E M T$ and MESSCS are not approximable within $O\left(\ln ^{2-\epsilon} n\right)$, unless $N P \subseteq Z T I M E\left(n^{\text {polylog }(n)}\right)$.

We now present a method for approximating MESSCS. Let $I_{M E S S C S}$ be an instance of $I_{M E S S C S}$ that consists of a complete directed graph $G=(V, E)$ with edge cost function $c: E \rightarrow R^{+}$and a set of terminals $D \subseteq V$. Pick an arbitrary node $v_{0} \in V$ and let $I_{M E M T}$ and $I_{M E I M T}$ be the instances of MEMT and MEIMT, respectively, consisting of the graph $G$ with edge cost function $c$, the root node $v_{0}$ and the set of terminals $D-\left\{v_{0}\right\}$.

Assume we have weight assignments $w_{1}$ and $w_{2}$ to the nodes of $V$ which are solutions for $I_{M E M T}$ and $I_{M E I M T}$, respectively. Construct the weight assignment $w_{3}$ defined as $w_{3}(u)=\max \left\{w_{1}(u), w_{2}(u)\right\}$ for every $u \in V$. We can prove that if the weight assignments $w_{1}$ and $w_{2}$ are $\rho_{1}-$ and $\rho_{2}-$ approximate solutions for $I_{M E M T}$ and $I_{M E I M T}$ respectively, then the weight assignment $w_{3}$ is a $\left(\rho_{1}+\rho_{2}\right)$-approximate solution to $I_{M E S S C S}$.

We can solve $I_{M E M T}$ and $I_{M E I M T}$ using the $O\left(|D|^{\epsilon}\right)$-approximation algorithm of Liang [14] and the $O\left(|D|^{\epsilon}\right)$-approximation algorithm of [5] for DST. In this way we obtain the following.

Theorem 5. For any $\epsilon>0$, there exists an $O\left(|D|^{\epsilon}\right)$-approximation algorithm for MESSCS.

\section{Logarithmic Approximations for MEBT and MESCS}

In this section we show that MEBT and MESCS can be approximated within a logarithmic factor. These results are optimal within constant factors. 
Liang in [14] presents an intuitive reduction for transforming an instance $I_{M E B T}$ of MEBT into an instance $I_{D S T}$ of DST in such a way that a $\rho$ approximate solution for $I_{D S T}$ implies a $\rho$-approximate solution for $I_{M E B T}$.

We describe this reduction here. Assume that $I_{M E B T}$ consists of a complete directed graph $G=(V, E)$ with an edge cost function $c: E \rightarrow R^{+}$and a root node $r \in V$. Then, the instance $I_{D S T}$ consists of a directed graph $H=(U, A)$ with an edge cost function $c^{\prime}: A \rightarrow R^{+}$, a root node $r^{\prime} \in U$ and a set of terminals $D \subseteq U-\left\{r^{\prime}\right\}$. For a node $v \in V$, we denote by $n_{v}$ the number of different edge costs in the edges directed out of $v$, and, for $i=1, \ldots, n_{v}$, we denote by $X_{i}(v)$ the $i$-th smallest edge cost among the edges directed out of $v$. For each node $v \in V$, the set of nodes $U$ contains $n_{v}+1$ nodes $Z_{v, 0}, Z_{v, 1}, \ldots, Z_{v, n_{v}}$. For each directed edge $(v, u) \in E$ and for $i=1, \ldots, n_{v}$, the set of edges $A$ contains a directed edge of zero cost from $Z_{v, i}$ to $Z_{u, 0}$ if $X_{i}(v) \geq c(v, u)$. Also, for each node $v \in V$, and $i=1, \ldots, n_{v}$, the set of edges $A$ contains a directed edge from $Z_{v, 0}$ to $Z_{v, i}$ of cost $c^{\prime}\left(Z_{v, 0}, Z_{v, i}\right)=X_{i}(v)$. The set of terminals is defined by $D=\left\{Z_{v, 0} \mid v \in V-\{r\}\right\}$ and $r^{\prime}=Z_{r, 0}$.

We use an algorithm proposed by Zosin and Khuller [17 to approximate $I_{D S T}$ by repeatedly solving instances of the Minimum Density Directed Tree (MDDT) problem. An instance of MDDT is defined in the same way as instances of DST and the objective is to compute a tree directed out of the root node such that the ratio of the cost of the tree over the number of terminals it spans is minimized. The algorithm of [17] repeatedly solves instances $I_{M D D T}^{i}$ of MDDT derived by the instance $I_{D S T}$. The instance $I_{M D D T}^{1}$ is defined by the graph $H$ with edge cost function $c$, the set of terminals $D_{1}=D$ and the root node $r_{1}=r^{\prime}$. Initially, the algorithm sets $i=1$. While $D_{i} \neq \emptyset$, it repeats the following. It finds a solution $T$ to $I_{M D D T}^{i}$ that consists of a tree $T_{i}=\left(V\left(T_{i}\right), E\left(T_{i}\right)\right)$, defines the instance $I_{M D D T}^{i+1}$ by contracting the nodes of $T_{i}$ into the root node $r_{i+1}$ and by setting $D_{i+1}=D_{i} \backslash V\left(T_{i}\right)$, and increments $i$ by 1 .

Zosin and Khuller [17] show that if the solution $T_{i}$ is a $\rho$-approximate solution for $I_{M D D T}^{i}$ in each iteration $i$, then the union of the trees $T_{i}$ computed in all iterations is an $O(\rho \ln n)$-approximate solution for $I_{D S T}$. They also show how to find a $(d+1)$-approximate solution for $I_{M D D T}^{i}$ if the graph obtained when removing the terminals from $G$ has depth $d$. Observe that, given an instance $I_{M E B T}$ of MEBT, the graph $H$ obtained by applying the reduction of Liang is bipartite, since there is no edge between nodes of $D \cup\left\{r^{\prime}\right\}$ and between nodes of $V-\left(D \cup\left\{r^{\prime}\right\}\right)$. Thus, the graph obtained by removing the terminals of $D$ from $H$ has depth 1 . Following the reasoning of [17], we obtain the following result.

Theorem 6. There exists an $O(\ln n)$-approximation algorithm for $M E B T$.

Now, following a similar technique with the one we used in section 5 for approximating MESSCS, we can solve any instance of MESCS by solving an instance of MEBT and an instance of MEIBT and then merging the two solutions. In this way we obtain an $O(\ln n)$-approximation algorithm for MESCS. 


\section{Approximating Bidirected MESS and Related Problems}

In the following, we present a logarithmic approximation algorithm for bidirected MESS. The algorithm uses a reduction from instances of bidirected MESS to instances of NWSF.

Consider an instance $I_{b M E S S}$ of bidirected MESS which consists of a complete directed graph $G=(V, E)$, an edge cost function $c: E \rightarrow R^{+}$and a set of terminals $D \subseteq V$ partitioned into $p$ disjoint subsets $D_{1}, D_{2}, \ldots, D_{p}$.

We construct an instance $I_{N W S F}$ of NWSF consisting of an undirected graph $H=(U, A)$, a node weight function $c^{\prime}: U \rightarrow R^{+}$and a set of terminals $D^{\prime} \subseteq U$ partitioned into $p$ disjoint sets $D_{1}^{\prime}, D_{2}^{\prime}, \ldots, D_{p}^{\prime}$. For a node $v \in V$, we denote by $n_{v}$ the number of different edge costs in the edges directed out of $v$, and, for $i=1, \ldots, n_{v}$, we denote by $X_{i}(v)$ the $i$-th smallest edge cost among the edges directed out of $v$. The set of nodes $U$ consists of $n$ disjoint sets of nodes called supernodes. Each supernode corresponds to a node of $V$. The supernode $Z_{v}$ corresponding to node $v \in V$ has the following $n_{v}+1$ nodes: a hub node $Z_{v, 0}$ and $n_{v}$ bridge nodes $Z_{v, 1}, \ldots, Z_{v, n_{v}}$. For each pair of nodes $u, v \in V$, the set of edges $A$ contains an edge between the bridge nodes $Z_{v, i}$ and $Z_{u, j}$ such that $X_{i}(u) \geq c(u, v)$ and $X_{j}(v) \geq c(v, u)$. Also, for each node $v \in V, A$ contains an edge between the hub node $Z_{v, 0}$ and each bridge node $Z_{v, i}$, for $i=1, \ldots, n_{v}$. The cost function $c^{\prime}$ is defined as $c^{\prime}\left(Z_{v, 0}\right)=0$ for the hub nodes and as $c^{\prime}\left(Z_{v, i}\right)=$ $X_{i}(v)$ for $i=1, \ldots, n_{v}$, for the bridge nodes. The set of terminals $D^{\prime}$ is defined as $D^{\prime}=\cup_{i} D_{i}^{\prime}$ where $D_{i}^{\prime}=\left\{Z_{v, 0} \in U \mid v \in D_{i}\right\}$.

Consider a subgraph $F=\left(S, A^{\prime}\right)$ of $H$ which is a solution for $I_{N W S F}$. We construct a weight assignment $w$ on the nodes of $G$ by setting $w(v)=0$ if $S$ contains no node from supernode $Z_{v}$, and $w(v)=\max _{u \in\left(Z_{v} \cap S\right)} c^{\prime}(u)$, otherwise. We can prove that if $F$ is a $\rho$-approximate solution to $I_{N W S F}$, then $w$ is a $\rho$-approximate solution to $I_{b M E S S}$.

In [11, Guha and Khuller present a $1.61 \ln k$-approximation algorithm for NWSF, where $k$ is the number of terminals in the graph. Using this algorithm for solving $I_{N W S F}$, we obtain a solution of $I_{b M E S S}$ which is within $1.61 \ln |D|$ of optimal. Moreover, when $p=1$ (i.e., when $I_{b M E S S}$ is actually an instance of bidirected MESSCS), the instance $I_{N W S F}$ is actually an instance of NWST which can be approximated within $1.35 \ln k$, where $k$ is the number of terminals in the graph [11]. The next theorem summarizes the discussion of this section.

Theorem 7. There exist an $1.61 \ln |D|$-, an $1.35 \ln |D|$-, and an $1.35 \ln n$ approximation algorithm for bidirected MESS, bidirected MESSCS, and bidirected MESCS.

\section{References}

1. E. Althaus, G. Călinescu, I. Măndoiu, S. Prasad, N. Tchervenski, and A. Zelikovsky. Power Efficient Range Assignment in Ad-Hoc Wireless Networks. In Proc. of the IEEE Wireless Communications and Networking Conference (WCNC '03), IEEE Computer Society Press, pp. 1889-1894, 2003. 
2. P. Berman and M. Karpinski. On Some Tighter Inapproximability Results. In Proc. of the 26th International Colloquium on Automata, Languages, and Programming (ICALP '99), LNCS 1644, Springer, pp. 200-209, 1999.

3. G. Călinescu, S. Kapoor, A. Olshevsky and A. Zelikovsky. Network Lifetime and Power Assignment in Ad-Hoc Wireless Networks. In Proc. of the 11th Annual European Symposium on Algorithms (ESA '03), 2003, to appear.

4. I. Caragiannis, C. Kaklamanis and P. Kanellopoulos. New Results for EnergyEfficient Broadcasting in Wireless Networks. In Proc. of the 13th Annual International Symposium on Algorithms and Computation (ISAAC '02), LNCS 2518, Springer, pp. 332-343, 2002.

5. M. Charikar, C. Chekuri, T.-Y. Cheung, Z. Dai, A. Goel, S. Guha and M. Li. Approximation Algorithms for Directed Steiner Problems. Journal of Algorithms, 33(1):73-91, 1999.

6. M. Chlebík and J. Chlebíková. Approximation Hardness of the Steiner Tree Problem on Graphs. In Proc of the 8th Scandinavian Workshop on Algorithm Theory (SWAT '02), LNCS 2368, Springer, pp. 170-179, 2002.

7. A. E. F. Clementi, P. Crescenzi, P. Penna, G. Rossi, and P. Vocca. On the Complexity of Computing Minimum Energy Consumption Broadcast Subgraphs. In Proc. of the 18th Annual Symposium on Theoretical Aspects of Computer Science (STACS '01), LNCS 2010, Springer, pp. 121-131, 2001.

8. A. E. F. Clementi, P. Penna, and R. Silvestri. Hardness Results for the Power Range Assignment Problem in Packet Radio Networks. In Proc. of Randomization, Approximation, and Combinatorial Optimization (RANDOM/APPROX '99), LNCS 1671, Springer, pp. 197-208, 1999.

9. J. Edmonds. Optimum Branchings. Journal of Research of the National Bureau of Standards, 71B:233-240, 1967.

10. M. X. Goemans and D. P. Williamson. A General Approximation Technique for Constrained Forest Problems. SIAM Journal on Computing, 24:296-317, 1995.

11. S. Guha and S. Khuller. Improved Methods for Approximating Node Weighted Steiner Trees and Connected Dominating Sets. Information and Computation, 150(1):57-74, 1999.

12. E. Halperin and R. Krauthgamer. Polylogarithmic Inapproximability. In Proc. of the 35th Annual ACM Symposium on Theory of Computing (STOC '03), pp. 585$594,2003$.

13. L. M. Kirousis, E. Kranakis, D. Krizanc, and A. Pelc. Power Consumption in Packet Radio Networks. Theoretical Computer Science, 243(1-2):289-305, 2000.

14. W. Liang. Constructing Minimum-Energy Broadcast Trees in Wireless Ad Hoc Networks. In Proc. of 3rd ACM International Symposium on Mobile Ad Hoc Networking and Computing (MOBIHOC '02), pp. 112-122, 2002.

15. G. Robins and A. Zelikovsky. Improved Steiner Tree Approximations in Graphs. In Proc. of the 11th Annual ACM-SIAM Symposium on Discrete Algorithms (SODA '00), pp. 770-779, 2000.

16. P.-J. Wan, G. Călinescu, X.-Y. Li, and O. Frieder. Minimum-Energy Broadcasting in Static Ad Hoc Wireless Networks. Wireless Networks, 8(6):607-617, 2002.

17. L. Zosin and S. Khuller. On Directed Steiner Trees. In Proc. of the 13th Annual ACM/SIAM Symposium on Discrete Algorithms (SODA '02), pp. 59-63, 2002. 